\title{
Os registos dos médicos de família estão em perigo
}

Mónica Granja*

\section{RESUMO}

Os registos médicos são uma peça chave da prestação de cuidados de saúde. Nos registos dos médicos de família portugueses encontram-se frequentemente dados base muito incompletos, a substituição de notas clínicas progressivas por itens da Classificação Internacional de Cuidados Primários, problemas que não são listados ao seu mais alto nível de resolução e listas de problemas que não servem como resumo completo e rigoroso do paciente. Limitações da aplicação informática «SClínico Cuidados de Saúde Primários» podem ser uma explicação para estes problemas com os registos. Esta aplicação, por um lado, não é compatível com o Registo Médico Orientado por Problemas; por outro, força ao uso da Classificação Internacional de Cuidados Primários antes do registo propriamente dito. Imposições na avaliação de desempenho das unidades de saúde são outra possível explicação. Os médicos de família precisam de investir na qualidade dos seus registos e de participar no desenvolvimento das respetivas aplicações informáticas, mantendo-os centrados no paciente e nos cuidados a prestar.

Palavras-chave: Medicina geral e familiar; Registos médicos; Registo Médico Orientado por Problemas; Registos clínicos eletrónicos

\section{INTRODUÇÃO}

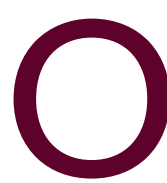

s registos médicos são uma peça chave da prestação de cuidados de saúde. Eles são, simultaneamente, o repositório da informação clínica de um paciente, um instrumento do processo de cuidados, um suporte do desenvolvimento da experiência clínica dos médicos, uma ferramenta de investigação, por um lado, e de gestão, por outro. ${ }^{1}$ Os registos são ainda uma peça chave nos processos médico-legais.

Na sua função de repositório, muito potenciada pelo formato eletrónico, é preciso obter uma solução de compromisso entre guardar o máximo de informação relevante e consegui-lo num formato lógico e sistemático que torne acessível e manuseável esse volume de informação. ${ }^{2}$ Para tal é fundamental que a estrutura dos registos seja arquitetada numa perspetiva global de sistemas de informação e que o seu desenvolvimento seja testado no terreno. ${ }^{3-4}$

Enquanto instrumento no processo de cuidados, os registos são, por um lado, «a memória do médico», ${ }^{5}$ par-

*Médica de família. Centro de Saúde de S. Mamede Infesta, Unidade Local de Saúde de Matosinhos.

Professora auxiliar convidada (Unidades Curriculares de Medicina Geral e Familiar), Instituto de Ciências Biomédicas Abel Salazar (Universidade do Porto). te da garantia da longitudinalidade dos cuidados, assegurando, por outro, a continuidade de cuidados entre diferentes profissionais e unidades de saúde. É, no entanto, necessário garantir que, em qualquer das suas funções, o ato de registar não interfira negativamente no próprio processo de cuidados. ${ }^{2,6}$ Em Portugal, as aplicações informáticas têm sido postas no terreno sem uma avaliação sistemática do seu impacto, mas existem estudos noutros países com resultados desfavoráveis. ${ }^{6}$

Os registos médicos são comparáveis às anotações que um cientista faz das suas observações: ${ }^{1}$ notas dispersas que, coligidas, integrarão essa espécie de big picture que é a experiência clínica, fonte insubstituível de aprendizagem. Nesta perspetiva, os registos tendem a ser centrados no médico e aspetos como a experiência de adoecer, as expectativas e os recursos tendem a ser subregistados e, logo, subvalorizados. ${ }^{1}$

Enquanto dados de investigação e informação base de políticas de gestão, os registos médicos são um manancial de informação de elevado potencial. No entanto, se não se aferir a sua validade, os registos resultarão inúteis, senão perigosos. Além disso, e no caso particular da medicina geral e familiar (MGF), deve ser mantido na mente de médicos, investigadores e gestores que mesmo os registos mais rigorosos não coinci- 
dem totalmente com a prática médica, existindo entre ambos um inexorável hiato, comparável ao que existe entre um mapa (os registos) e o território real (a prática). É que é nesse hiato que reside a essência da MGF que tem muito de inefável, de incognoscível e de imen-

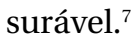

\section{Problemas com registos médicos}

Os registos médicos em suporte eletrónico generalizaram-se nos centros de saúde portugueses nos últimos dez anos. Hoje os médicos de família (MF) acedem facilmente a registos feitos por outros colegas em todas as zonas do país e noutros níveis de cuidados. O acesso direto e bidirecional à informação clínica de pacientes cuidados por diferentes médicos e em diferentes locais é uma mais-valia potencial ao serviço da continuidade de cuidados, característica primordial da MGF. Mas, na prática, essa mais-valia só se concretiza se os registos forem de qualidade (inteligíveis, rigorosos e completos). Lamentavelmente constata-se que, em muitos casos, quer nas notas clínicas progressivas SOAP quer nas listas de problemas, o texto livre foi parcial ou integralmente substituído por itens e códigos da Classificação Internacional de Cuidados Primários (ICPC), ${ }^{8}$ transformando registos num «repositório seco» de códigos onde é difícil apurar seja a história da doença atual, seja o resumo dos principais problemas do paciente. ${ }^{5}$ Um segundo problema é a dificuldade em registar e encontrar itens importantes dos dados base do paciente, como perceber com quem vive, a sua história profissional, os seus recursos e apoios ou mesmo como gostaria de ser designado pelos profissionais.

\section{Substituição dos registos por itens da ICPC}

Porque é que os registos dos MF estão a ser substituídos por itens da classificação ICPC? Existem pressões diretas e indiretas. A pressão indireta vigora há mais tempo e é exercida através da arquitetura da aplicação informática usada na maioria dos centros de saúde (CS): o «SClínico Cuidados de Saúde Primários» (SClínico CSP) e o seu percursor, o «SAM Centro de Saúde». Desde o início da sua utilização, há mais de 10 anos, que o SAM/SClínico CSP força a classificação: para inserir um problema na lista de problemas é necessário classificá-lo. E é suficiente classificá-lo, pelo que a maioria das vezes os problemas são listados apenas pelo respe- tivo item da ICPC. Apesar de existir um campo para texto livre após este item, a sua utilização é muito pouco amigável.* Além disso, alguns itens da ICPC-2 aparecem automaticamente no $\mathbf{A}$ e no $\mathbf{P}$ do SOAP quando se abrem programas de saúde, quando se fazem certos registos, se se referencia aos cuidados hospitalares ou emite uma guia de tratamento. A codificação forçada $\mathrm{e}$ o desincentivo à inserção de texto livre ameaçam a precisão do registo. ${ }^{9}$

As pressões diretas são mais recentes. Em 2014, o Ministério da Saúde publicou uma circular normativa indicando que os MF "devem classificar todos os problemas abordados na consulta e manter a lista de problemas atualizada com os mais importantes". ${ }^{10}$ Há indicadores de desempenho que premeiam determinadas classificações no A (e.g., em pacientes obesos ou com consumos nocivos). ${ }^{11}$ E, apesar de em 2014 o próprio Ministério da Saúde ter admitido que classificar motivos de consulta e procedimentos não é útil na prática clínica diária, ainda há pelo menos um critério de avaliação em vigor que premeia uma classificação no P. ${ }^{12}$

Nesta substituição dos registos por itens de uma classificação, patente no SOAP e nas listas de problemas, parece ser esquecido que classificar é diferente de registar. ${ }^{8}$ Por um lado, nem todos os componentes do RMOP são classificáveis. ${ }^{13}$ No $\mathbf{S}$ apenas se classificam motivos de consulta, não existindo qualquer sistema que classifique outros sinais e sintomas apresentados pelo paciente ou despertados pelo próprio médico. No $\mathbf{O}$ apenas são classificáveis os procedimentos realizados, mas não o seu resultado. No A não é possível classificar hipóteses de diagnóstico ou diagnósticos diferenciais. No $\mathbf{P}$ não são classificáveis, entre outros, os acordos estabelecidos com o paciente, os planos de longo prazo e outros lembretes para consultas seguintes.

\footnotetext{
* Apesar de ser um espaço com muitos caracteres disponíveis, apenas são visíveis 30 de cada vez, e minúsculos. Durante o registo, o cursor está constantemente a regressar ao primeiro destes caracteres. A título de exemplo, a descrição de problemas comuns como «diabetes tipo 2 insulinotratada desde 2003 atualmente controlada sem hipoglicémias complicada de microalbuminúria» ou «adenocarcinoma alto grau T2N1M0 mama esq a mastectomia + esvaziamento axilar 2009 (IPO) + radioterapia x 2 meses + hormonoterapia entre 2009-14 + reconstrução mamária 2011 HSJoão» atinge facilmente os 100 caracteres.
} 
Classificar serve parte das funções dos registos médicos (gestão de cuidados e investigação), mas não traz qualquer vantagem às funções de repositório individual e de instrumento no processo individual de cuidados. ${ }^{8}$ Por outro lado, classificar é abstrair num caso clínico singular os aspetos comuns a outros casos, ${ }^{8}$ perdendo-se detalhes cruciais como a caracterização dos sintomas, a sua evolução ou a negação da presença de sintomas relevantes no quadro em causa. Ao substituir o A por itens da ICPC perde-se a avaliação que o MF faz dos problemas da consulta como, por exemplo, a sua gravidade, evolução e grau de controlo. Igualmente ao substituir o $\mathbf{P}$ pelos itens da IPC perde-se a informação sobre o plano educacional, diagnóstico e terapêutico concretamente acordado com o paciente.

Quanto à lista de problemas, a substituição dos registos por itens da ICPC resulta em perda de detalhe em alguns casos e em excesso de detalhe noutros. Esta incongruência subverte a hierarquização dos problemas e impede o elencar de cada problema ao mais alto nível de resolução. Por exemplo, para listar o problema «diabetes tipo 2 controlado com antidiabéticos orais e complicado por retinopatia diabética» é necessário selecionar dois itens ICPC (como se fossem dois problemas e não um só): «diabetes não insulinodependente» e «retinopatia» (que inclui todos os tipos de retinopatia). Já para registar o problema «patologia músculo-esquelética degenerativa generalizada» dispomos de pelo menos 10 itens, a que se podem associar mais uns quantos em caso de agudização ou traumatismo. Este tipo de limitações esteve na base dos resultados de um estudo norueguês que concluiu que a ICPC não era adequada para o trabalho clínico e que deveria ponderar-se uma solução alternativa. ${ }^{14}$

Provavelmente a maioria dos MF portugueses não é suficientemente competente no uso da ICPC. ${ }^{15}$ Estudos noutros países têm sugerido que os sistemas de classificação existentes promovem mais heterogeneidade que consistência. ${ }^{14}$

Ainda na lista de problemas, a substituição dos registos por itens da ICPC resulta em problemas enunciados com nomenclatura desadequada e fora de uso, como é exemplo flagrante o caso da diabetes que é subdividida em insulinodependente (correspondendo à diabetes tipo 1) e não insulinodependente (correspondendo à diabetes tipo $2 \mathrm{e}$ à diabetes secundária).
O SClínico CSP possibilita que se complemente a classificação ICPC dos problemas com a codificação CID-10 (10ª Revisão da Classificação Estatística Internacional de Doenças e Problemas Relacionados com a Saúde), possibilitando uma melhor definição de alguns casos, mas deixando por definir muitos problemas dos pacientes.

\section{Outros problemas do SClínico CSP}

O SClínico CSP é uma aplicação insuficiente e pouco compatível face a dois dos três componentes do RMOP: a lista de problemas e os dados base.

Como atrás exposto, por ser obrigatoriamente estruturada a partir da ICPC, frequentemente não é possível construir uma lista de problemas adequada. Mas a lista de problemas do SClínico CSP apresenta outras limitações. Exige que seja indicado para cada problema um dia preciso para o seu início, o que é impossível de fazer com rigor para muitos problemas (e.g., os diagnosticados décadas atrás, anteriormente a qualquer registo disponível, informático ou outro). A respeito de datas, de referir ainda a transição abrupta entre o processo de papel e o eletrónico, na maioria dos casos sem transporte de dados, originando listas de problemas em que, não importando a idade do paciente, quase só há problemas com início na última década.

Ainda na lista de problemas, o SClínico CSP permite que sejam incluídos itens da ICPC que não são problemas (e.g., «sem doença» ou «Medicina preventiva e de acompanhamento geral»). Além disso, insere automaticamente alguns itens na lista (e.g., «contraceção oral» quando se abre o programa de planeamento familiar e se outro método não for selecionado, mesmo que seja um homem ou uma mulher que não use método algum). Não é claro quem definiu a inserção automática destes itens.

O outro componente do RMOP que enfrenta problemas de incompatibilidade com o SClínico CSP são os dados base. Falta espaço para registo de alguns dados, outros encontram-se dispersos por diferentes janelas e atalhos da aplicação e outros ainda estão formatados rigidamente, não permitindo incorporar a sua diversidade nem a sua dinâmica. ${ }^{15}$

Por exemplo, não há nenhum local para registo de composição real do agregado familiar (tantas vezes não coincidente com o agregado «administrativo» regista- 
do), com possibilidade de notação da sua dinâmica (divórcios, mortes, saídas e regresso de filhos). Não há também possibilidade de registo (ou anexação) do genograma, da história familiar e social, dos hábitos de exercício físico, dos cuidadores, recursos e apoios dos pacientes mais dependentes, das migrações, sequer do nome ou título pelo qual o paciente prefere ser tratado.

Exemplo de rigidez é o espaço para registo da profissão, impedindo o registo de uma história profissional. O espaço obriga ao uso da Classificação Portuguesa das Profissões onde boa parte das profissões dos pacientes não se encontra facilmente. A versão atual desta classificação (2010) inclui mais de 700 profissões diferentes e o respetivo manual de utilização tem quase 500 páginas. ${ }^{16}$ Além disso, não permite registar profissões passadas, duração de eventuais exposições de risco, nem descrever funções específicas (devido aos escassos caracteres disponíveis para texto livre).

A história reprodutiva das mulheres é um exemplo de registo disperso por dois programas de saúde (planeamento familiar e saúde materna) e apenas para as mulheres que estão em algum destes programas.

Também a medicação crónica e passada dos pacientes se encontra apenas parcialmente disponível: pode ser consultada no SClínico CSP (com longo tempo de espera e fechando as restantes janelas), mas só pode ser alterada na PEM (plataforma de prescrição eletrónica) que abre numa janela diferente e sujeita a diferente autenticação. Em caso de referenciação não é possível importar a medicação crónica para a carta de referenciação.

Outro exemplo de rigidez e dispersão é o espaço para registo das características da habitação: uma checklist acessível a partir da agenda, com nove itens e sem possibilidade de inserção de texto livre. Também os antecedentes familiares estão disponíveis numa janela anexa à lista de problemas onde, como na própria lista de problemas, só se pode inserir um antecedente, classificando-o à cabeça, não importando quão vago ele seja referido pelo paciente.

Por fim, o SClínico CSP não é uma aplicação estável nem fiável. Ocorrem com frequência atualizações que perturbam ou mesmo impedem o seu funcionamento durante inúmeras consultas de MGF. Ocasionalmente, estas atualizações levam à perda de dados: em 2017 perderam-se os registos de profissões e contactos de $e$-mail dos pacientes colhidos pelos MF duramente uma década.

\section{Oportunidades e ameaças}

As potenciais vantagens dos registos eletrónicos são muito valiosas. McWhinney listava uma dezena, ${ }^{1}$ a maioria das quais permanece por alcançar na MGF portuguesa: organização dos cuidados preventivos com alertas automáticos para médicos e pacientes; acesso imediato a resultados de exames e à lista de medicação crónica; alertas automáticos sobre interações medicamentosas, alergias e contraindicações; ferramentas de apoio ao diagnóstico diferencial e à decisão clínica; ajuda no seguimento de doentes crónicos com geração de alertas para gestos em falta; integração horizontal (com cuidados domiciliários e outros agentes da comunidade) e vertical (com cuidados hospitalares) da informação clínica; ${ }^{\dagger}$ integração automática de resultados de exames; possibilidade de anexar notas de alta, relatórios, fotografias em formato digital; e possibilidade de uso de conversores de voz em texto escrito. A estas vantagens inacessíveis, acrescem ainda a possibilidade de incorporação automática de mensagens de $e$-mail e o registo em tempo real de telefonemas com pacientes uma realidade já possível em alguns países através de plataformas seguras. O motor do desenvolvimento do SClínico CSP parece ser a voracidade por dados e pelo controlo da produtividade médica e não a melhoria da prestação de cuidados ou a segurança dos pacientes.

Os MF devem estar alerta para os potenciais prejuízos dos registos médicos eletrónicos. Aplicações que forçam a classificação e desincentivam o texto livre põem em risco a precisão dos registos ${ }^{9} \mathrm{e}$ desvalorizam aspetos chave da MGF como a capacidade de lidar com a incerteza e a perspetiva holística. ${ }^{1}$ A perturbação da relação médico-paciente pode acontecer quando a aplicação requer muita atenção por parte do MF, como acontece quando é necessário procurar ícones pequenos, abrir e fechar sucessivas janelas, preencher grandes listas de dados ou aguardar pelo avanço de aplicações lentas. A organização por «programas de saúde» imposta pelo SClínico CSP e a impossibilidade de man-

\footnotetext{
† A Plataforma de Dados de Saúde proporciona um somatório de informação do eixo vertical, mas sem integração da informação num documento único.
} 
ter a maioria das suas inúmeras janelas abertas em simultâneo fragmenta a informação e perturba a visão global do paciente. Esta visão global, além de central na MGF, é cada vez mais necessária com a crescente multimorbilidade decorrente do envelhecimento populacional e do aumento da esperança de vida.

McWhinney alertava para as consequências nem sempre previsíveis do uso das tecnologias. ${ }^{1}$ Avisava que a possibilidade de escrutínio que os registos eletrónicos permitem a gestores e governantes poderia introduzir conflitos de interesse nas decisões clínicas. Achava especialmente perigosa a ideia de os registos eletrónicos serem transformados numa tecnologia com autoridade própria. É possível que isso já esteja a acontecer na MGF portuguesa.

\section{Propostas}

A aplicação informática a utilizar para registos pelos MF tem de ser compatível com o RMOP (ou com o método de registo que a cada momento venha a ser considerado pela MGF como o mais adequado). A estrutura da lista de problemas deve ser reformulada para comportar a listagem de problemas enunciados ao mais alto nível de resolução e com informação sobre estadio, grau de controlo, sequelas, marcos de evolução e de tratamento. Os dados base devem ser agrupados num único espaço onde, não obstante possam existir lembretes, a informação possa ser inserida e atualizada em texto livre flexível, espelhando a dinâmica pessoal, familiar, social e laboral do paciente. A divisão dos registos em programas de saúde deve ceder lugar a um programa único que permita uma visão holística do paciente e permita escolher para cada paciente os parâmetros mais relevantes. A medicação crónica registada deve ser incorporada no registo da consulta com possibilidade de transposição automática para as cartas de referenciação.

O desenvolvimento de uma aplicação informática compatível com o RMOP deve envolver MF, cujo foco seja a prestação de cuidados de saúde centrados na pessoa. ${ }^{4}$ Os MF no terreno devem ter papel decisor nas sucessivas atualizações a introduzir nas aplicações informáticas de registo, inclusive naquelas cujo objetivo seja responder a necessidades de gestão e não servir diretamente a prestação de cuidados. O desenvolvimento de aplicações informáticas para registos médicos deve ser um processo em que diferentes soluções são testadas no terreno antes de generalizadas, de modo a assegurar que modelos conceptualmente bons são aplicáveis e trazem mais benefícios que prejuízos na prática clínica diária. ${ }^{4,6}$

A fiabilidade do manancial de dados disponível por via dos registos médicos eletrónicos tem de ser avaliada. A qualidade dos registos médicos deve ser auditada, nomeadamente quanto à sua correspondência com o conteúdo da consulta, completude e consistência. $\mathrm{O}$ uso da ICPC a partir de registos inexistentes ou ininteligíveis não é passível de avaliação e deverá ser rejeitado.

Para aspetos menos padronizados dos registos, como, por exemplo, que problemas incluir na lista de problemas, devem ser definidas políticas locais com envolvimento dos MF no terreno.

Os registos automáticos e os forçados devem ser eliminados. O uso da ICPC para possibilitar a obtenção de dados para gestão e investigação não deve ter prioridade sobre a primeira função do MF e dos registos que é a prestação de cuidados médicos de boa qualidade. Para maximizar a classificação sugere-se o uso de janelas com sugestões geradas por ferramentas de reconhecimento de texto, que o MF possa optar por aceitar ou não. ${ }^{9}$ Para obter classificações de qualidade, uma alternativa a considerar é o uso de MF classificadores em diferido da consulta. À semelhança do que acontece nos hospitais, estes classificadores, com treino específico e ferramentas adequadas de reconhecimento de texto, classificariam os registos dos MF no terreno, podendo funcionar, em simultâneo, como auditores da qualidade dos registos.

Por fim, a apresentação gráfica da aplicação deve ser amigável, tão estável quanto possível e em questões de dimensões (de ícones, letras e espaços em geral) ter em conta o intervalo de idades dos médicos (entre os $25 \mathrm{e}$ os 67 anos).

\section{CONCLUSÃO}

Os registos dos MF não têm acompanhado o caminho de qualidade que a MGF portuguesa tem feito. Entre as possíveis causas estão limitações com a aplicação informática mais usada (o SClínico CSP) que não é compatível com o RMOP. Outra causa possível é a substituição dos registos pelo uso da ICPC, uso este força- 
do pelo SClínico CSP e imposto na avaliação de desempenho das unidades de saúde. Os MF precisam de investir na qualidade dos seus registos e de participar no desenvolvimento das respetivas aplicações informáticas, mantendo-os centrados no paciente e nos cuidados a prestar.

\section{AGRADECIMENTOS}

À Dra. Conceição Outeirinho, médica de família e regente das Unidades Curriculares de Medicina Geral e Familiar do Mestrado Integrado de Medicina, do Instituto de Ciências Biomédicas Abel Salazar, pela revisão crítica do manuscrito.

\section{REFERÊNCIAS BIBLIOGRÁFICAS}

1. McWhinney IR, Freeman T. Records. In: McWhinney IR, Freeman T, editors. A textbook of family medicine. 3rd ed. New York: Oxford University Press; 2009. p. 375-8.

2. Caeiro RT. Registos clínicos em medicina familiar. Lisboa: Instituto de Clínica Geral da Zona Sul; 1991.

3. Prieto Orzanco A, Juncosa Font S, Alonso López F. Los sistemas de información. In: Casado Vicente V, Calero Muñoz S, Cordón Granados F, Ezquerra Lezcano M, García Olmos L, García Velasco G, et al, editors. Tratado de medicina de familia y comunitaria. $2^{\mathrm{a}}$ ed. Barcelona: semFYC; 2012. p. 287-310. ISBN 9788498355857

4. Høstgaard AM, Bertelsen P, Nøhr C. Methods to identify, study and understand end-user participation in HIT development. BMC Med Inform Dec Mak. 2011;11:57.

5. Braga R. Os registos clínicos e a codificação [Clinical records and coding]. Rev Port Med Geral Fam. 2012;28(3):155-6. Portuguese

6. Bossen C. Evaluation of a computerized problem-oriented medical record in a hospital department: does it support daily clinical practice? Int J Med Inform. 2007;76(8):592-600.

7. Heath I. That by which it is what it is. Br J Gen Pract. 2009;59(562):e1423.

8. Pinto D. Classificar motivos de consulta e procedimentos com a ICPC na prática clínica? [Classifying reasons for encounter and procedures with ICPC in clinical practice?]. Rev Port Med Geral Fam. 2012;28(4):247-8. Portuguese

9. Simons SM, Cillessen FH, Hazelzet JA. Determinants of a successful problem list to support the implementation of the problem-oriented medical record according to recent literature. BMC Med Inform Decis Mak. 2016;16:102.
10. Administração Central dos Sistemas de Saúde. Recomendações para a utilização da Classificação Internacional de Cuidados de Saúde Primários (ICPC) na prática clínica diária em Unidades do SNS: circular normativa n. ${ }^{\circ}$ 20/2014/DPS/ACSS, de 16-07-2014 [Internet]. Lisboa: Ministério da Saúde; 2017 [cited 2017 Feb 4]. Availlable from: http:// www.acss.min-saude.pt/circulares/Circular_Normativa/2014/Circular_Normativa_20_2014.pdf

11. Administração Central dos Sistemas de Saúde. Bilhete de identidade dos indicadores de contratualização dos Cuidados de Saúde Primários para o ano de 2016 [Internet]. Lisboa: Ministério da Saúde; 2016 [cited 2017 Feb 4]. Available from: http://www.acss.min-saude.pt/wpcontent/uploads/2016/07/bilhete_identidade_indicadores_contratualizacao_2016__2016_02_16.pdf

12. Administração Central dos Sistemas de Saúde. Guia para a aplicação do diagnóstico do desenvolvimento organizacional nas USF - DiOr-USF [Internet]. Lisboa: Ministério da Saúde; 2012 [cited 2017 Feb 4]. Available from: http://www2.acss.min-saude.pt/Portals/0/Guia\%20DiOrUSF.pdf

13. Pinto D. O que classificar nos registos clínicos com a Classificação Internacional de Cuidados Primários? [What should we code in health records with the International Classification of Primary Care?]. Rev Port Med Geral Fam. 2014;30(5):328-34. Portuguese

14. Botsis T, Bassøe CF, Hartvigsen G. Sixteen years of ICPC use in Norwegian primary care: looking through the facts. BMC Med Inform Decis Mak. 2010;10:11.

15. Melo M. O uso da ICPC nos registos clínicos em medicina geral e familiar [The use of ICPC in clinical records in general practice]. Rev Port Med Geral Fam. 2012;28(4):245-6. Portuguese

16. Queiroz MJ. SOAP revisitado [SOAP revisited]. Rev Port Clin Geral. 2009;25(2):221-7. Portuguese

17. Instituto Nacional de Estatística. Classificação portuguesa das profissões 2010. Lisboa: INE; 2011. ISBN 9789892500102

\section{CONFLITOS DE INTERESSE}

O autor declara não ter quaisquer conflitos de interesse.

\section{ENDEREÇO PARA CORRESPONDÊNCIA}

Mónica Granja

E-mail: monicagranja66@gmail.com

Recebido em 26-02-2017

Aceite para publicação em 10-01-2018 


\section{ABSTRACT}

\section{FAMILY PRACTICE RECORDS ARE IN DANGER}

Medical records are a key element of health care delivery. Records written by Portuguese family physicians often contain incomplete baseline information, progressive replacement of clinical notes by items of the International Classification of Primary Care, problems that are not listed according to their highest level of resolution and lists of problems that are neither complete nor a rigorous clinical summary of the patient. Limitations of the software 'SClínico Cuidados de Saúde Primários' may explain these problems on medical records. This application is not compatible with the Problem-Oriented Medical Record; on the other hand, it forces the use of the International Classification of Primary Care before the entry of actual clinical notes. Imposed performance indicators may also be another possible explanation. Family physicians must persist in the quality of their records and take an active part in the development of electronic health record systems, keeping them centred on the patient and on health care delivery.

Keywords: Family practice; Medical records; Problem-oriented medical records; Electronic health records. 